\title{
LTE-V for Sidelink 5G V2X Vehicular Communications: A New 5G Technology for Short-Range Vehicle-to- Everything Communications
}

\author{
RafaelMolnaMasegosaand Javier Gozalvez UniversidadMiguel HemandezdeEche(UMH)
}

This paper provides an overview of the LTE-V standard supporting sidelink or V2V communications using the PC5 interface in LTE. The paper reviews the physical layer changes introduced under Release 14 for LTE-V, its communication modes 3 and 4, and the LTE-V evolutions under discussion in Release 15 to support $5 G \quad V 2 X$ communications and autonomous vehicles' applications. Modes 3 and 4 support direct V2V communications, but differ on how they allocate the radio resources. Resources are allocated by the cellular network under Mode 3. Mode 4 does not require cellular coverage, and vehicles autonomously select their radio resources using a distributed scheduling scheme supported by congestion control mechanisms. Mode 4 is considered the baseline mode, and represents an alternative to $802.11 p$ or DSRC. In this context, the paper also presents a detailed analysis of the performance of LTE-V sidelink Mode 4, and proposes a modification to its distributed scheduling.

\section{Introduction}

V2X (Vehicle-to-Everything) communications will enable the exchange of information between vehicles (Vehicle to Vehicle, V2V), and between vehicles and other nodes (infrastructure and pedestrians). This exchange will provide vehicles with a more accurate knowledge of their surrounding environment that can improve the traffic safety [1]. Important efforts have been devoted over the last years to develop V2X communications using IEEE 802.11p. However, 802.11p uses a CSMA/CA medium access scheme, and can face some challenges to guarantee strict reliability levels and ensure the network's scalability as the load increases [2]. As an alternative, the Third Generation Partnership Project (3GPP) published in September 2016 the first version of Release 14 that includes support for V2X communications [3]. The standard is commonly referred to as LTE-V, LTE-V2X or Cellular V2X. The LTE-V physical layer improves the link budget with regards to $802.11 p$. In addition, LTE-V can increase the reliability, under certain conditions, by adding a redundant transmission per packet. The LTE-V standard includes two radio interfaces. The $\mathrm{Uu}$ interface supports
Vehicle to Infrastructure (V2I) communications, while the PC5 interface supports V2V communications based on direct LTE sidelink. LTE sidelink or D2D (Device to Device) was introduced for the first time under Release 12 for public safety, and includes two modes of operation: Mode 1 and Mode 2. Both modes were designed with the objective to prolong the battery lifetime of mobile devices at the cost of increasing the latency. Connected vehicles require highly reliable and low latency V2X communications, and therefore Mode 1 and 2 are not suitable for vehicular applications. Release 14 introduces two new communication modes (Mode 3 and Mode 4) specifically designed for V2V communications. In Mode 3, the cellular network selects and manages the radio resources used by vehicles for their direct V2V communications. In Mode 4, vehicles autonomously select the radio resources for their direct V2V communications. Mode 4 can hence operate without cellular coverage, and is therefore considered the baseline V2V mode since safety applications cannot depend on the availability of cellular coverage. Mode 4 includes a distributed scheduling scheme for vehicles to select their radio resources, and the support for distributed congestion control.

This paper presents an overview of LTE-V sidelink V2V communications based on the PC5 interface in Release 14 (referred to as LTE-V in the rest of the paper). The tutorial includes a review of the LTE-V physical layer, and presents in detail Mode 3 and Mode 4 for V2V communications. In addition, the paper reviews the support for congestion control in LTE-V, and the enhancements that are currently under discussion in Release 15. Mode 4 is the baseline mode for V2V communications using LTE-V. As a result, the paper includes a comprehensive simulation evaluation of the performance of LTE-V Mode 4. Based on the results of this analysis, the authors propose a modification to its distributed scheduling.

\section{Physical Layer}

LTE-V utilizes SC-FDMA, and supports 10 and $20 \mathrm{MHz}$ channels. Each channel is divided into sub-frames, Resource Blocks (RBs), and sub-channels. Sub-frames are 1ms long (like the Transmission Time Interval). A RB is the smallest unit of frequency resources that can be allocated to a user. It is 
$180 \mathrm{kHz}$ wide in frequency (12 sub-carriers of $15 \mathrm{kHz}$ ). LTE-V defines sub-channels as a group of RBs in the same sub-frame. The number of RBs per sub-channel can vary. Sub-channels are used to transmit data and control information. The data is transmitted in Transport Blocks (TBs) over Physical Sidelink Shared Channels (PSSCH), and the control information in Sidelink Control Information (SCI) messages over Physical Sidelink Control Channels (PSCCH) [4]. A TB contains a full packet to be transmitted, e.g. a beacon or Cooperative Awareness Message (CAM). A node that wants to transmit a TB must also transmit its associated SCI (also referred to as SA Scheduling Assignment). The SCI includes information such as the modulation and coding scheme used to transmit the TB, the RBs it uses, and the resource reservation interval for semipersistent scheduling. This information is critical for other nodes to be able to receive and decode the transmitted TB, so the SCI must be correctly received. A TB and its associated SCI must always be transmitted in the same sub-frame. We refer to the transmission of an SCI and its associated TB in the same sub-frame as SCI+TB (or HARQ transmission in 3GPP). LTE-V defines 2 sub-channelization schemes (Figure 1):

- Adjacent PSCCH+PSSCH. The SCI and TB are transmitted in adjacent RBs. For each SCI+TB transmission, the SCI occupies the first two RBs of the first sub-channel utilized for the transmission. The TB is transmitted in the RBs following the SCI, and can occupy several sub-channels (depending on its size). If it does so, it will also occupy the first two RBs of the following sub-channels.

- Non-Adjacent PSCCH+PSSCH. The RBs are divided into pools. One pool is dedicated to transmit only SCIs, and the SCIs occupy 2 RBs. The second pool is reserved to transmit only TBs and is divided into sub-channels.

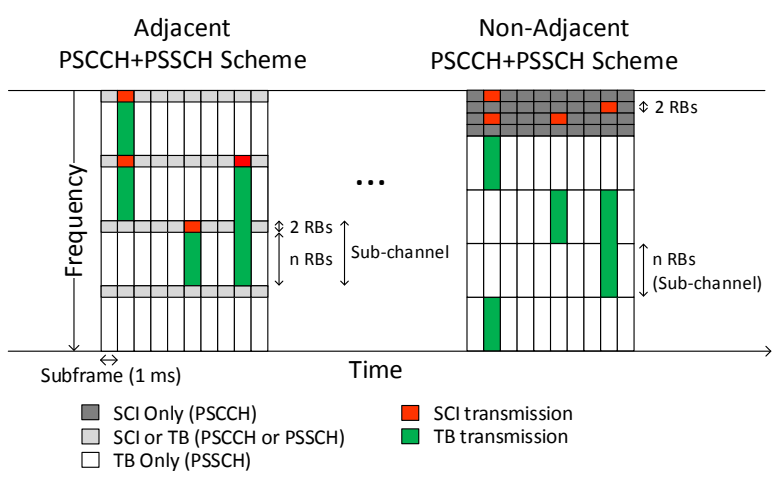

Figure 1 LTE-V sub-channelization

TBs can be transmitted using QPSK or 16-QAM, whereas the SCIs are always transmitted using QPSK. LTE-V uses turbo coding and normal cyclic prefix. LTE-V sub-carriers have a total of 14 symbols per sub-frame. Four of these symbols are dedicated to the transmission of Demodulation Reference Signals (DMRS) in order to combat the Doppler effect at high speeds. DMRSs are transmitted in the $3^{\text {rd }}, 6^{\text {th }}, 9^{\text {th }}$ and $12^{\text {th }}$ symbol of each sub-carrier per sub-frame [5]. The maximum transmit power is $23 \mathrm{dBm}$, and the standards specify a sensitivity power level requirement at the receiver of $90.4 \mathrm{dBm}$ and a maximum input level of $-22 \mathrm{dBm}[6]$.

\section{Mode 4}

Vehicles communicate using sidelink or V2V communications under Mode 4, and autonomously select their radio resources independently of whether they are under cellular coverage or not. When the vehicles are under cellular coverage, the network decides how to configure the V2X channel and informs the vehicles through the Sidelink V2X Configurable Parameters [3]. The message includes, among others: the carrier frequency of the V2X channel, the V2X resource pool, synchronization references, the subchannelization scheme, the number of sub-channels per subframe, and the number of RBs per sub-channel. When the vehicles are not under cellular coverage, they utilize a preconfigured set of parameters in replacement of the Sidelink V2X Configurable Parameters. However, the standard does not specify a concrete value for each parameter. The V2X resource pool indicates which sub-frames of a channel are utilized for V2X. The rest of sub-frames can be utilized by other services (including cellular communications). The standard includes the option to divide the V2X resource pool based on geographical areas (referred to as Zoning [3]). In this case, vehicles in an area can only utilize the pool of resources that have been assigned to such area. We will assume in this paper that a channel is completely dedicated to $\mathrm{V} 2 \mathrm{X}$ and that Zoning is not applied.

\section{Sensing-based Semi-Persistent Scheduling}

Vehicles select their sub-channels in Mode 4 using the sensing-based Semi-Persistent Scheduling (SPS) scheme specified in Release 14 [4][7]. A vehicle reserves the selected sub-channel(s) for a number of consecutive Reselection Counter packet transmissions. This counter is randomly set between 5 and 15, and the vehicle includes its value in the SCI. After each transmission, the Reselection Counter is decremented by one. When it is equal to zero, new resources must be selected and reserved with probability $(1-P)$. Each vehicle can set up $P$ between 0 and 0.8 . New resources need to be reserved also if the packet to be transmitted does not fit in the sub-channel(s) previously reserved. The Reselection Counter is randomly chosen every time new resources must be reserved. Packets can be transmitted every 100 sub-frames (i.e. 10 packets per second or 10pps) or in multiples of 100 subframes (up to a minimum of 1pps). Each vehicle includes its packet transmission interval in the resource reservation field of its SCI. Thanks to the semi-persistent reservation of resources, and the inclusion of the Reselection Counter and packet transmission interval in the SCI, other vehicles can estimate which sub-channels are free when making their own reservation, which reduces packet collisions. The process to reserve sub-channels is organized in the following three steps. 
Step 1. Let's suppose that a vehicle $V$ needs to reserve new subchannels at time $T$. It can reserve sub-channels between $T$ and the established maximum latency (equal or lower than $100 \mathrm{~ms}$ [4]). This time period is referred to as selection window. Within the selection window, the vehicle identifies as Candidate Single-Subframe Resources (CSRs) ${ }^{1}$ to be reserved, all groups of adjacent sub-channels within the same sub-frame where the SCI+TB to be transmitted fits.

Step 2. Vehicle $V$ analyzes all the information it has received in the 1000 sub-frames before $T$, and creates a list $L_{l}$ of CSRs it could reserve. This list includes all the CSRs in the selection window except those that meet the following two conditions:

1) $V$ has correctly received in the last 1000 subframes an SCI from another vehicle indicating that it will utilize this CSR at the same time as $V$ will need it to transmit any of its next Reselection Counter packets;

2) $V$ measures an average Reference Signal Received Power (RSRP) over the RBs utilized to transmit the TB associated to the SCI higher than a given threshold ${ }^{2}$.

The two conditions must be simultaneously met in order for $V$ to exclude a CSR. Vehicle $V$ also excludes all CSRs of subframe $F$ in the selection window, if $V$ was transmitting during any previous sub-frame $F-100 * j(\mathrm{j} \in \mathrm{N}, 1 \leq \mathrm{j} \leq 10)^{3}$.

After Step 2 is executed, $L_{1}$ must include at least $20 \%$ of all CSRs in the selection window. If not, Step 2 is iteratively executed until the $20 \%$ target is met. The RSRP threshold is increased by $3 \mathrm{~dB}$ in each iteration.

Step 3. Vehicle $V$ creates a second list $L_{2}$ of CSRs. The total number of CSRs in $L_{2}$ must be equal to $20 \%$ of all CSRs in the selection window. $L_{2}$ includes the CSRs from $L_{1}$ (after Step 2) that experienced the lowest average RSSI (Received Signal Strength Indicator) over all its RBs. This RSSI value is averaged over all the previous $T_{C S R}-100 * j$ sub-frames $(j \in \mathrm{N}, 1$ $\leq \mathrm{j} \leq 10$ ), see Figure 2 . Vehicle $V$ randomly chooses one of the CSRs in $L_{2}$, and reserves it for the next Reselection Counter packet transmissions.

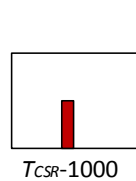

(ms)

Average sensed RSSI $=\frac{\sum_{j=1}^{10} \operatorname{RSSI}\{T C S R-100 * j\}}{10}$

Figure 2 Step 3: average RSSI of a candidate resource

\footnotetext{
${ }^{1}$ Also referred to as Candidate Resources.

2 The threshold depends on the priority of the packet. This priority is established by higher layers based on the relevance and urgency of the application. If $V$ receives several SCIs from the same interfering vehicle reserving a given CSR, it will utilize the most recent one to estimate the average RSRP.

${ }^{3} V$ is not able to receive the transmissions of other vehicles in the subframe it is transmitting (half-duplex transmissions).
}

\section{Extensions to sensing-based SPS}

LTE-V Mode 4 provides the option for each packet to be transmitted twice to increase the reliability. In this case, the sensing-based SPS scheme creates a third list $L_{3}$ of CSRs. Let's suppose that the original SCI+TB transmission took place in a CSR in sub-frame $S F . L_{3}$ is made of all CSRs included in $L_{2}$ (produced in Step 3) that are located in the time interval [SF$15 \mathrm{~ms} ; S F+15 \mathrm{~ms}$ ], with the exception of all the CSRs in $S F$. The sensing-based SPS scheme randomly selects a CSR from $L_{3}$ for the redundant transmission of the SCI+TB. The selection for redundant transmissions is maintained semi-persistently for the following Reselection Counter packets.

The sensing-based SPS scheme can support higher packet transmission frequencies than 10pps, in particular 20pps and 50pps. In this case, the following changes to the sensing-based SPS scheme are applied:

- The maximum tolerable latency is $50 \mathrm{~ms}$ and $20 \mathrm{~ms}$ respectively, which reduces the selection window.

- The Reselection Counter can take any value between 10 and 30 for 20pps, and between 25 and 75 for 50pps.

- The variable $j$ in Step 3 takes values between 1 and 20 for 20pps, and between 1 and 50 for 50pps.

Release 14 includes a variant of the sensing-based SPS scheme for Pedestrian to Vehicle communications, where pedestrians broadcast their presence using mobile devices. The sensing process seriously compromises the battery of these devices, so the standard gives them the option to only sense a percentage of the 1000 sub-frames previous to $T$ in Step 2. The mobile devices can only select (using the sensing-based SPS scheme) CSRs in the sensed sub-frames.

\section{Mode 3}

Vehicles also communicate using sidelink or V2V communications under Mode 3. However, the selection of subchannels is managed by the base station or eNB, and not by each vehicle as it is the case in Mode 4. Mode 3 is hence only available when vehicles are under cellular coverage. 3GPP has defined the necessary cellular architecture enhancements to support V2X. One of these enhancements is the V2X Control Function that is used by the network in Mode 3 to manage radio resources and to provide vehicles (or in general UEs) with the Sidelink V2X Configurable Parameters. Mode 3 utilizes the same sub-channel arrangements as defined for Mode 4. Vehicles using Mode 3 must also transmit an associated SCI per TB, and the transmission of the SCI and TB must take place in the same sub-frame. Differently from Mode 4, the standards do not specify a resource management algorithm for Mode 3. Each operator can implement its own algorithm that should fall under one of these two categories [8]:

- Dynamic Scheduling. Vehicles request sub-channels to the eNB for each packet transmission. This increases the cellular signaling overhead, and delays the packet transmission until vehicles are notified of their assigned sub-channels. 
- Semi-persistent Scheduling (SPS). The eNB reserves subchannels for the periodic transmissions of a vehicle like in Mode 4. However, differently from Mode 4, it is up to the eNB to decide for how long should the reservation be maintained (i.e. Mode 3 does not define a Reselection Counter). Only the eNB can activate, de-activate or modify a reservation of sub-channels for a vehicle. The vehicle must inform the eNB of the size, priority and transmission frequency of its packets so that the eNB can semipersistently reserve the appropriate sub-channels. This information (referred to as UE Assistance Information [4]) must be provided to the eNB at the start of a transmission, or when any of the traffic characteristics (size, priority and frequency) change.

Vehicles operating under Mode 3 can be supported by different cellular operators or PLMNs (Public Land Mobile Networks). To enable their direct communications, 3GPP has defined an inter-PLMN architecture [9] that can support the following scenarios:

- Vehicles supported by different PLMNs transmit in different carriers. In this case, vehicles must be able to simultaneously receive in multiple carriers to receive the transmissions of vehicles supported by other PLMNs. To this aim, each PLMN broadcasts in the Sidelink V2X Configurable Parameters the necessary information so that the vehicles it supports can receive the packets transmitted by vehicles supported by other PLMNs.

- Vehicles supported by different PLMNs share the same carrier, but each PLMN is assigned part of the RBs of the carrier. The standard does not specify how the resources should be split among the PLMNs, but introduces a coordination mechanism (through the V2X Control Function) between PLMNs to avoid packet collisions.

\section{Congestion Control}

Release 14 supports congestion control in Mode 4 [3]. The standard does not specify a particular congestion control algorithm, but defines the related metrics and possible mechanisms to reduce the channel congestion. Each time a vehicle has to transmit or retransmit a packet, it estimates the Channel Busy Ratio (CBR) and Channel occupancy Ratio (CR). If the packet is going to be transmitted at sub-frame $n$, the measurements are done at sub-frame $n-4$ [4]. The CBR provides an indication of the level of channel congestion, and is defined as the amount of sub-channels in the previous 100 subframes that experience an average RSSI higher than a preconfigured threshold ${ }^{4}$. The $\mathrm{CR}$ quantifies the channel occupancy generated by the transmitting vehicle. It is defined as the amount of sub-channels that the transmitting vehicle utilizes during a period of 1000 sub-frames. This period can

${ }^{4}$ The standard does not specify this threshold, but 3GPP working documents usually compute this threshold by adding $-107 \mathrm{dBm}$ per RB in the sub-channel. include past and future ${ }^{5}$ sub-frames, and it is up to each vehicle to decide how many past and future sub-frames it takes into account when computing the $\mathrm{CR}$ with certain restrictions. In particular, the CR must be estimated taking into account at least the previous 500 sub-frames, and only the future subframes that are already reserved by the transmitting vehicle can be considered to compute the CR.

The standard indicates that up to sixteen CBR intervals can be defined, and for each one, a transmitting vehicle cannot overpass a maximum $\mathrm{CR}_{\text {Limit }}$ that augments as the CBR decreases. The value of $\mathrm{CR}_{\mathrm{Limit}}$ for each $\mathrm{CBR}$ interval varies with the priority of the packet. The standard does not specify the range of each CBR interval and the values of $\mathrm{CR}_{\text {Limit. }}$ However, Table 1 shows an example from 3GPP working documents [10] for $10 \mathrm{pps}^{6}$.

\begin{tabular}{|c|c|}
\hline CBR measured & CR $_{\text {Limit }}$ \\
\hline $\mathrm{CBR} \leq 0.65$ & no limit \\
\hline $0.65<\mathrm{CBR} \leq 0.675$ & $1.6 \mathrm{e}-3$ \\
\hline $0.675<\mathrm{CBR} \leq 0.7$ & $1.5 \mathrm{e}-3$ \\
\hline $0.7<\mathrm{CBR} \leq 0.725$ & $1.4 \mathrm{e}-3$ \\
\hline $0.725<\mathrm{CBR} \leq 0.75$ & $1.3 \mathrm{e}-3$ \\
\hline $0.75<\mathrm{CBR} \leq 0.775$ & $1.2 \mathrm{e}-3$ \\
\hline $0.8<\mathrm{CBR} \leq 0.825$ & $1.1 \mathrm{e}-3$ \\
\hline $0.825<\mathrm{CBR} \leq 0.85$ & $1.1 \mathrm{e}-3$ \\
\hline $0.85<\mathrm{CBR} \leq 0.875$ & $1.0 \mathrm{e}-3$ \\
\hline $0.875<\mathrm{CBR}$ & $0.8 \mathrm{e}-3$ \\
\hline
\end{tabular}

Table 1 Example of CBR intervals and $C_{\text {Limit }}$ for congestion control under LTE-V mode 4 [10].

When a vehicle has to transmit a packet (or its redundant version), it measures the $\mathrm{CBR}$ and quantifies its $\mathrm{CR}$. If its $\mathrm{CR}$ is higher than the value of $\mathrm{CR}_{\text {Limit }}$ specified for the CBR interval that includes the measured $\mathrm{CBR}$, the vehicle must reduce its $\mathrm{CR}$ below $\mathrm{CR}_{\text {Limit. }}$ To do so, the standard defines several possible mechanisms [7][8], and it is up to each vehicle to decide which one to utilize:

- Packet dropping. The vehicle reduces its CR by not transmitting certain packets generated by the application (but maintains the reserved sub-channels).

- Number of transmissions per packet. The vehicle can reduce its $\mathrm{CR}$ by transmitting each packet only once (i.e. avoiding redundant transmissions).

- Modulation and coding scheme (MCS). The vehicle can reduce the $\mathrm{CR}$ by augmenting the MCS. This is possible if the pre-configured sub-channelization and the initial MCS results in that the transmission of a packet requires the use of various sub-channels. In this case, a packet can be transmitted using a lower number of sub-channels by increasing the MCS, which will in turn reduce the CR.

- Sub-channels reserved. A vehicle can reduce its CR by reducing the number of sub-channels it reserves per

\footnotetext{
${ }^{5}$ Future sub-frames can be taken into account as each vehicle reserves a number of sub-channels using the sensing-based SPS scheme.

${ }^{6}$ Higher transmission frequencies augment the $\mathrm{CR}$ within a period of 1000 sub-frames. In this case, the values of $\mathrm{CR}_{\text {Limit }}$ for each $\mathrm{CBR}$ interval should be re-visited.
} 
transmission. This can be achieved, for example, by augmenting the MCS that reduces the number of RBs necessary to transmit a packet.

- Transmission power. Decreasing the transmission power reduces the $\mathrm{CBR}$. If the resulting $\mathrm{CBR}$ level falls under a lower CBR interval, $\mathrm{CR}_{\text {Limit }}$ increases. In this case, a vehicle might be able to satisfy the condition that the $C R$ is below the $\mathrm{CR}_{\text {Limit }}$ without directly decreasing its $\mathrm{CR}$.

A vehicle can only modify the number of transmissions per packet or the number of reserved sub-channels when it has to execute the sensing-based SPS scheme to reserve new subchannels. The other mechanisms can be applied at any point in time, and on a per packet basis if needed. It is though important to emphasize that none of the congestion control mechanisms should force a new reservation of sub-channels. Forcing a new reservation before the Reselection Counter is equal to zero can negatively impact other vehicles that reserved their subchannels considering that surrounding vehicles would maintain their reservations. This negative impact is actually also present when implementing packet dropping. However, [11] showed that packet dropping improves the PDR (Packet Delivery Ratio) under congested channels.

Mode 3 does not implement a distributed congestion control process as defined in Mode 4. In Mode 3, the eNB manages the sub-channels, and decides how to reduce the channel occupancy. Such decisions can take into account the CBR levels locally measured by vehicles. To this aim, the eNB can request each vehicle to periodically (the eNB determines the period), or on-demand, report its measured CBR [8].

\section{Progress of Standardization Activities on 5G V2X}

3GPP has started the work on new 5G V2X enhancements under Release 15, and has completed the analysis of new use cases and requirements that will be supported by Release 15 [12]. Release 14 supports connected vehicle use cases such as forward collision warning. Release 15 new use cases are more focused on autonomous driving, and include: platooning, sensor and map sharing, information sharing for partial/conditional and high/full automated driving, and remote driving, among others. These applications can require the transmission of up to 50pps, a maximum latency between 3 and $10 \mathrm{~ms}$, and up to a $99.99 \%$ reliability level (defined in terms of PDR). To support these requirements, some of the LTE-V enhancements (referred to as V2X Phase 2 or eV2X) under discussion in Release 15 include [13]:

- Carrier Aggregation. LTE supports Carrier Aggregation up to 32 carriers. $3 \mathrm{GPP}$ is considering the aggregation of up to 8 carriers for LTE-V sidelink.

- 64-QAM modulation. Release 14 supports QPSK and 16QAM. 64-QAM increases the data rate and can reduce the channel occupancy. 3GPP is currently analyzing the need for a new DMRS scheme when introducing 64-QAM.
- Reduction of the maximum time between the packet arrives at layer 1 and the start of the selected sub-channel for transmission. Release 15 seeks to reduce this maximum time from 20ms (Release 14) to less than 10ms.

- Shared resources between Modes 3 and 4. Both modes could independently operate using a different pool of RBs. However, Release 15 is analyzing the possibility for both modes to coexist in order to optimize the usage of resources. Such coexistence might require changes for both modes. To date, only minor changes have been discussed, e.g. giving higher priority to Mode 3 reservations (indicated through the SCI), or including the resource reservation field in the SCI of Mode 3 transmissions so that Mode 4 can take them into account [14].

- Transmit Diversity. Release 15 is looking into the feasibility and gains that can be achieved in LTE-V using Transmit Diversity schemes (e.g. space time and frequency block coding, and small delay cyclic delay diversity).

- Transmission Time Interval (TTI). Release 15 is analyzing the possibility to reduce the TTI for LTE-V from $1 \mathrm{~ms}$ to $0.5 \mathrm{~ms}$ or 2 symbols. The analysis must take into account that vehicles with different TTI values (Release 14 and Release 15) will need to coexist in the same pool of RBs. Release 14 vehicles will not be able to overhear Release 15 vehicles using lower TTIs. However, their transmissions should not interfere with those from Release 14.

\section{Performance of LTE-V Mode 4}

This section presents a comprehensive analysis of the performance of LTE-V Mode 4 under the Highway Fast (60 vehicles per $\mathrm{km}$ at $140 \mathrm{~km} / \mathrm{h}$ ) and Highway Slow (120 vehicles per $\mathrm{km}$ at $70 \mathrm{~km} / \mathrm{h}$ ) scenarios defined in [15]. Vehicles transmit packets of 190bytes, except one of every five packets that has a size of 300bytes [15]. Packets are periodically transmitted every 100,50 or $20 \mathrm{~ms}(10,20$ or $50 \mathrm{pps}$ respectively). We assume that a $10 \mathrm{MHz}$ channel at $5.9 \mathrm{GHz}$ is completely dedicated to Mode 4 using Adjacent PSCCH+PSSCH subchannelization. Four sub-channels (of 12RBs each) are defined per sub-frame. The 300 bytes packets occupy two subchannels, and the 190 bytes packets one ${ }^{7} P$ is set equal to 0 . The propagation is modeled using the WINNER+ B1 pathloss model and a log-normal shadowing with spatial correlation. Radio transmission errors are modeled using Look-Up Tables (LUT) from [16] that map the SINR (Signal to Interference Noise Ratio) to the BLER (Block Error Rate). We assume perfect synchronization, and all vehicles transmit at $23 \mathrm{dBm}$. The noise figure is set to $9 \mathrm{~dB}$ and the RSRP threshold for the sensing-based SPS scheme is initially set to $-110 \mathrm{dBm}$.

Figure 3 compares the PDR of 802.11p and LTE-V as a function of the distance between transmitter and receiver. LTE-

\footnotetext{
${ }^{7}$ QPSK and a code rate of 0.5 is used for the TBs corresponding to the 300bytes packets (TBs occupy $20 \mathrm{RBs}$ ), whereas the 190bytes are transmitted with QPSK and a code rate of 0.7 (TBs occupy $10 \mathrm{RBs).}$
} 
$\mathrm{V}$ is configured without redundant transmissions, and vehicles transmit 10pps or 50pps. The figure shows that LTE-V outperforms 802.11p when 802.11p is configured with the default $6 \mathrm{Mbps}$ data rate. However, $802.11 \mathrm{p}$ can improve its performance when configured with a $18 \mathrm{Mbps}$ data rate [17], and even outperform LTE-V. We will later show that LTE-V can also increase its performance under highly loaded scenarios by augmenting the MCS. Figure 3 shows that when vehicles transmit 10pps, $802.11 \mathrm{p}$ can achieve a performance close to LTE-V up to a distance of $160 \mathrm{~m}$ if the $802.11 \mathrm{p}$ data rate is increased to $18 \mathrm{Mbps}$. From this distance, 802.11p achieves a smaller PDR than LTE-V due to the PHY layer performance and the dominant effect of propagation. When the channel load increases (vehicles transmit 50pps), packet collisions become the dominant source of errors. In this case, 802.11p can outperform LTE-V (with its configuration under analysis) if the $802.11 \mathrm{p}$ data rate is increased to $18 \mathrm{Mbps}$. As demonstrated in [17], augmenting the data rate reduces the channel load and packet collisions. The reduction of packet collisions under highly loaded scenarios (like when vehicles transmit 50pps) compensates the negative physical layer effects of increasing the data rate and using a less robust modulation and coding rate.

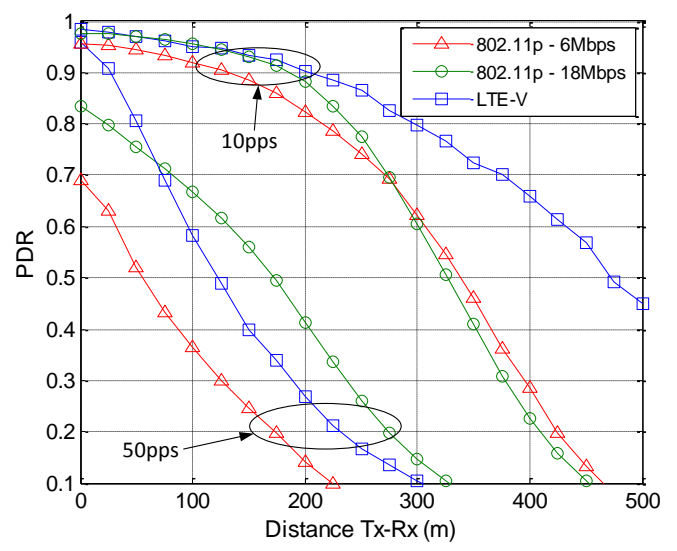

Figure 3 Comparison of LTE-V and 802.11p in Highway Slow.

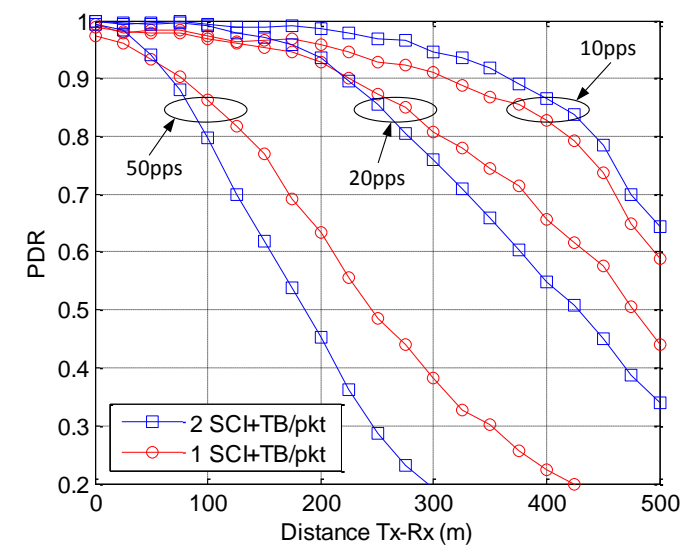

Figure 4 Effect of the number of transmissions per packet under the Highway Fast scenario.
Figure 3 shows that, just like 802.11p, the performance of LTE-V is significantly degraded when vehicles transmit more packets per second and the load increases. Many autonomous driving applications require vehicles to transmit a high number of packets per second. In this case, it is of interest to analyze the effectiveness of some of the congestion control mechanisms. Figure 4 shows the effect of transmitting each SCI+TB packet once (no redundancy) or twice. Figure 4 shows that redundant transmissions improve the PDR under low loads (10pps). However, redundant transmissions increase the load, and therefore the packet collisions as illustrated in Table 2 . This results in that redundant transmissions decrease the PDR from a certain distance when the load augments (Figure 4). However, redundant transmissions always improve the PDR at short distances between the transmitter and receiver due to the impact of Half-Duplex (HD) errors. These errors occur when a vehicle is transmitting in a sub-frame, and cannot receive the packets of other vehicles transmitting in the same sub-frame.

\begin{tabular}{|c|c|c|c|c|}
\hline Scenario & $\begin{array}{c}\text { Packets } \\
\text { per second }\end{array}$ & $\begin{array}{c}\text { Redundant } \\
\text { transmissi } \\
\text { on }\end{array}$ & $\begin{array}{c}\% \text { of } \\
\text { occupied } \\
\text { sub- } \\
\text { channels }\end{array}$ & $\begin{array}{c}\% \text { of sub- } \\
\text { channels } \\
\text { with } \\
\text { packet } \\
\text { collisions }\end{array}$ \\
\hline \multirow{6}{*}{$\begin{array}{c}\text { Highway } \\
\text { Fast }\end{array}$} & \multirow{2}{*}{10} & No & $17.08 \%$ & $0.78 \%$ \\
\hline & & Yes & $31.72 \%$ & $3.21 \%$ \\
\hline & \multirow{2}{*}{20} & No & $32.22 \%$ & $2.76 \%$ \\
\hline & & Yes & $55.03 \%$ & $14.37 \%$ \\
\hline & \multirow{2}{*}{50} & No & $62.08 \%$ & $23.33 \%$ \\
\hline & & Yes & $80.39 \%$ & $56.83 \%$ \\
\hline \multirow{6}{*}{$\begin{array}{l}\text { Highway } \\
\text { Slow }\end{array}$} & \multirow{2}{*}{10} & No & $32.46 \%$ & $3.38 \%$ \\
\hline & & Yes & $55.23 \%$ & $14.13 \%$ \\
\hline & \multirow{2}{*}{20} & No & $55.05 \%$ & $14.53 \%$ \\
\hline & & Yes & $76.09 \%$ & $44.47 \%$ \\
\hline & \multirow{2}{*}{50} & No & $80.91 \%$ & $56.64 \%$ \\
\hline & & Yes & $91.35 \%$ & $79.05 \%$ \\
\hline
\end{tabular}

Table 2 Impact of redundant transmissions and number of packets transmitted per second on packet collisions.

The probability of HD errors is independent of the distance and non-negligible when each SCI+TB packet is transmitted only once and vehicles transmit a high number of packets per second (e.g. it is equal to $2.5 \%$ for $50 \mathrm{pps}$ ). Redundant transmissions eliminate HD errors since the probability that two vehicles transmit their two SCI+TB packets in the same sub-frame is negligible.

[17] showed that increasing the data rate can improve the performance of $802.11 \mathrm{p}$, especially when channels are congested. Figure 5 analyzes the effect of the data rate on the LTE-V performance considering that all packets have a size of 190 bytes, and that each packet is transmitted twice. The figure depicts the PDR for QPSK and a code rate of 0.5 (QPSK-r0.5), QPSK and a code rate of 0.7 (QPSK-r0.7), and 16QAM and a code rate of 0.5 (16QAM-r0.5). Each packet occupies 16, 12 and 8 RBs with QPSK-r0.5, QPSK-r0.7 and 16QAM-r0.5, and each sub-frame includes 3,4 and 6 sub-channels respectively. Increasing the data rate decreases the error protection, but also 
the number of packet collisions (Table 3). The lower number of collisions explains why increasing the data rate always improves the PDR under the Highway Slow scenario (Figure 5). This effect is not always observed in Highway Fast where vehicles move at faster speeds $(140 \mathrm{~km} / \mathrm{h})$. In this case, the Doppler effect has a significant impact on the link level performance of 16QAM-r0.5 that exhibits an error floor [16] (cannot reduce the BLER below 8\% even for high SINRs). The lower packet collisions obtained with 16QAM-r0.5 does not compensate this error floor when vehicles transmit 20pps (Figure 5.a). However, when the load increases (50pps), 16QAM-r0.5 significantly reduces the packet collisions (Table 3), and achieves the best PDR despite its error floor (Figure 5.b).

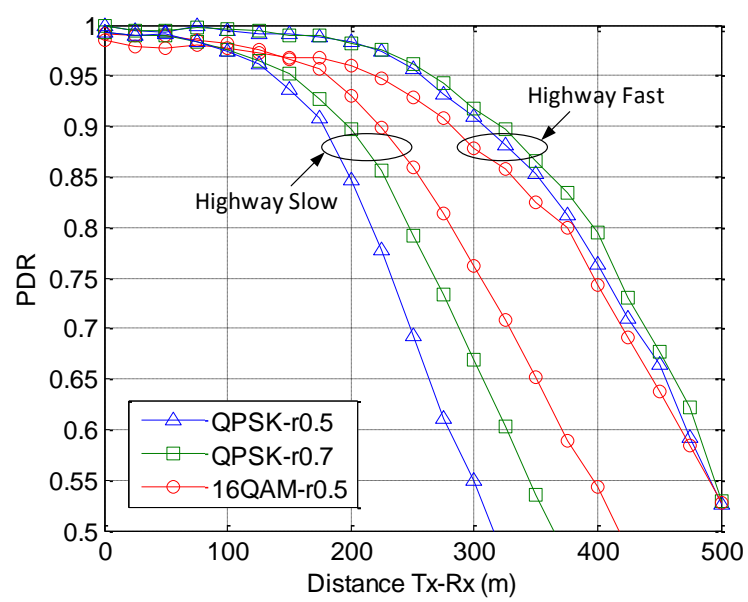

(a) 20pps

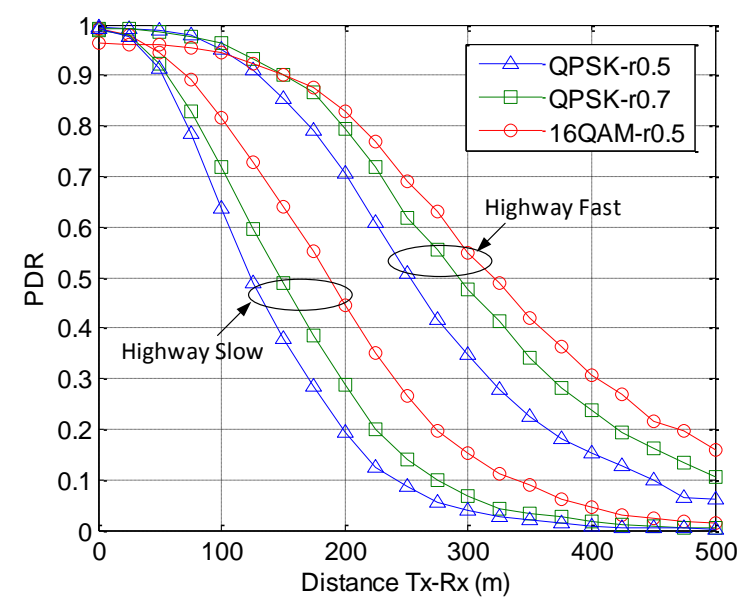

(b) $50 \mathrm{pps}$

Figure 5 Effect of the MCS.

\section{Modified sensing-based SPS}

The sensing-based SPS scheme can present certain inefficiencies when packets have different sizes and need a different number of sub-channels. This is the case with the traffic model in [15]. The baseline configuration utilized in the previous section results in that 300 bytes packets require two sub-channels, and 190bytes packets only one. A reservation done for a 190bytes packet will not be maintained for the following Reselection Counter transmissions since a 300bytes packet will be generated before the counter is equal to 0 . A new reservation will then be required for the 300 bytes packet, and the two reserved sub-channels will be maintained for Reselection Counter transmissions. This is highly inefficient since the following four transmissions correspond to 190bytes packets, and they only need one sub-channel. This results in that the sensing-based SPS scheme excludes more resources in Step 2 that are really being utilized, and more vehicles will compete for the non-excluded resources. To overcome this inefficiency, this paper proposes a modification to the sensingbased SPS scheme when the packets to be transmitted have different sizes, and the larger packets are less frequent than the smaller ones (likely scenario in vehicular communications). In particular, we propose that no sub-channels are reserved when transmitting the larger packets (300bytes in this study). The sensing-based SPS scheme is used to select the sub-channels necessary to transmit this packet. However, the selected subchannels will not be reserved for the following Reselection Counter transmissions. Instead, the sensing-based SPS scheme will be again applied to select the sub-channel used to transmit the next 190bytes packet. This sub-channel will be the one reserved for the following Reselection Counter transmissions.

\begin{tabular}{|c|c|c|c|c|}
\hline Scenario & $\begin{array}{c}\text { Packets } \\
\text { per } \\
\text { second }\end{array}$ & MCS & $\begin{array}{c}\% \text { of } \\
\text { occupied } \\
\text { sub- } \\
\text { channels }\end{array}$ & $\begin{array}{c}\text { \% of sub- } \\
\text { channels } \\
\text { with } \\
\text { packet } \\
\text { collisions }\end{array}$ \\
\hline \multirow{6}{*}{$\begin{array}{c}\text { Highway } \\
\text { Fast }\end{array}$} & \multirow{3}{*}{20} & QPSK-r0.5 & $66.43 \%$ & $12.53 \%$ \\
\hline & & QPSK-r0.7 & $51.93 \%$ & $7.12 \%$ \\
\hline & & 16QAM-r0.5 & $36.88 \%$ & $2.54 \%$ \\
\hline & \multirow{3}{*}{50} & QPSK-r0.5 & $97.19 \%$ & $71.85 \%$ \\
\hline & & QPSK-r0.7 & $90.41 \%$ & $49.23 \%$ \\
\hline & & 16QAM-r0.5 & $78.57 \%$ & $20.56 \%$ \\
\hline \multirow{6}{*}{$\begin{array}{l}\text { Highway } \\
\text { Slow }\end{array}$} & \multirow{3}{*}{20} & QPSK-r0.5 & $91.82 \%$ & $51.82 \%$ \\
\hline & & QPSK-r0.7 & $82.28 \%$ & $29.82 \%$ \\
\hline & & 16QAM-r0.5 & $64.49 \%$ & $12.59 \%$ \\
\hline & \multirow{3}{*}{50} & QPSK-r0.5 & $99.82 \%$ & $97.05 \%$ \\
\hline & & QPSK-r0.7 & $99.13 \%$ & $90.22 \%$ \\
\hline & & 16QAM-r0.5 & $95.49 \%$ & $67.26 \%$ \\
\hline
\end{tabular}

Table 3. Impact of the MCS on channel occupancy and packet collisions. 


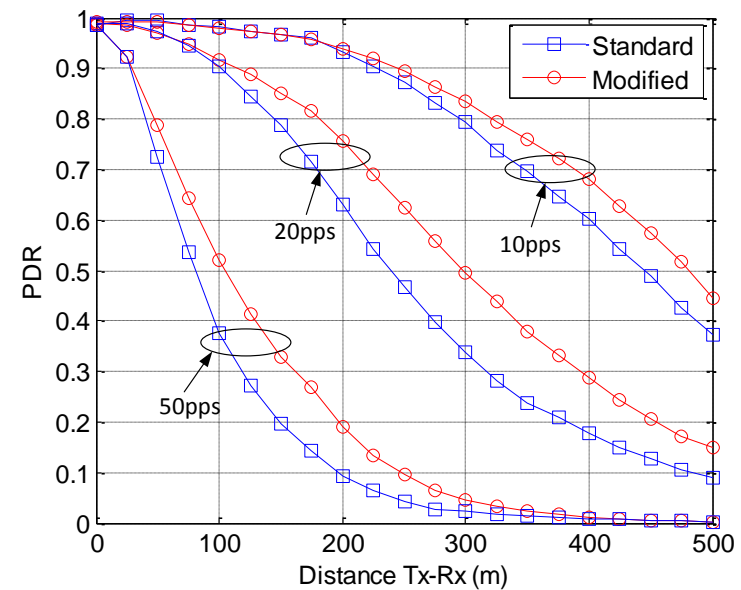

Figure 6 Comparison of the standard and modified sensingbased SPS (Highway Slow and redundant transmissions).

Figure 6 shows that the modified scheme improves the PDR compared to when applying the standard one defined in Release 14. The gains achieved are particularly relevant for medium to large distances and when vehicles transmit more packets per second. The gains achieved result from a more efficient utilization of sub-channels. Table 4 shows that vehicles utilize all the sub-channels they reserve with the modified scheme. On the other hand, the standard scheme results in that a large percentage of reserved sub-channels are not really utilized by the vehicles that reserved them. These unused sub-channels cannot be occupied by other vehicles, and the standard scheme occupies a lower percentage of subchannels than our proposal. This has a negative effect on the PDR of the standard scheme since vehicles compete for a smaller number of sub-channels, and therefore experience more packet collisions.

\begin{tabular}{|c|c|c|c|}
\hline \multirow{2}{*}{ Scheme } & $\begin{array}{c}\text { Packets } \\
\text { per second }\end{array}$ & $\begin{array}{c}\text { \% of occupied } \\
\text { sub-channels }\end{array}$ & $\begin{array}{c}\text { \% of reserved sub- } \\
\text { channels that are } \\
\text { utilized }\end{array}$ \\
\hline \multirow{3}{*}{ Standard } & 10 & $55.97 \%$ & $68.12 \%$ \\
\cline { 2 - 4 } & 20 & $77.07 \%$ & $64.79 \%$ \\
\hline \multirow{3}{*}{ Modified } & 50 & $92.04 \%$ & $62.32 \%$ \\
\cline { 2 - 4 } & 10 & $56.79 \%$ & $100 \%$ \\
\cline { 2 - 4 } & 20 & $85.01 \%$ & $100 \%$ \\
\hline
\end{tabular}

Table 4 Utilization of sub-channels by the standard and modified sensing-based SPS schemes (Highway Slow scenario and redundant transmissions).

\section{Conclusions}

This paper has presented a comprehensive overview of the LTE-V standard for sidelink or V2V communications based on the PC5 interface. The paper also includes a detailed analysis of LTE-V Mode 4. This mode is considered the baseline scheme as it does not require any cellular infrastructure support. The conducted study has shown that LTE-V can represent an alternative to $802.11 \mathrm{p}$ or DSRC due to its improved link budget, the support for redundant transmissions per packet, different sub-channelization schemes, and the infrastructure assistance under Mode 3. However, the distributed scheduling designed for LTE-V Mode 4 is not collision free, and requires a careful configuration of the transmission parameters, in particular for autonomous applications that require vehicles to transmit more packets per second. In this case, congestion control mechanisms and more efficient distributed scheduling schemes are necessary.

\section{References}

[1] M. Eiza, T. Owens, Q. Ni and Q. Shi, "Situation-Aware QoS Routing Algorithm for Vehicular Ad hoc Networks," IEEE Transactions on Vehicular Technology, Vol. 64, Issue 12, pp. 5520 - 5535, Dec 2015.

[2] G. Araniti, C. Campolo, M. Condoluci, A. Iera and A. Molinaro, "LTE for Vehicular Networking: A Survey," IEEE Communications Magazine, Volume: 51, Issue: 5, May. 2013.

[3] 3GPP, "TS 36.300 Evolved Universal Terrestrial Radio Access (E-UTRA) and Evolved Universal Terrestrial Radio Access Network (E-UTRAN); Overall description; Stage 2 (v14.3.0, Release 14)," 3GPP, Tech. Rep., Jun. 2017.

[4] 3GPP, "TS 36.213 Evolved Universal Terrestrial Radio Access (E-UTRA); Physical layer procedures (v14.3.0, Release 14)," 3GPP, Tech. Rep., Jun. 2017.

[5] 3GPP, "TS 36.211 Evolved Universal Terrestrial Radio Access (E-UTRA); Physical channels and modulation (v14.3.0, Release 14)," 3GPP, Tech. Rep., Jun. 2017.

[6] 3GPP, "TS 36.101 Evolved Universal Terrestrial Radio Access (E-UTRA); User Equipment (UE) radio transmission and reception (v14.4.0, Release 14)," 3GPP, Tech. Rep., Jul. 2017.

[7] 3GPP, “TS 36.321 Evolved Universal Terrestrial Radio Access (E-UTRA); Medium Access Control (MAC) protocol specification (v14.3.0, Release 14)," 3GPP, Tech. Rep., Jun. 2017.

[8] 3GPP, “TS 36.331 Evolved Universal Terrestrial Radio Access (E-UTRA); Radio Resource Control (RRC); Protocol specification (v14.3.0, Release 14),"3GPP, Tech. Rep., Jul. 2017.

[9] 3GPP, "TS 23.285 Architecture enhancements for V2X services (v14.3.0, Release 14)," 3GPP, Tech. Rep., Jun. 2017.

[10] Qualcomm Incorporated, "R1-1611594. Congestion control for V2V," 3GPP TSG RAN WG1 Meeting \#87, Reno, U.S.A., Nov. 2016.

[11] Intel Corporation, "R1-1702143. Evaluation of congestion control schemes for V2V communication," 3GPP TSG RAN WG1 Meeting \#88, Athens, Greece, Feb. 2017.

[12] 3GPP, "TR 22.886 Study on enhancement of 3GPP support for 5G V2X services (v15.0.0, Release 15)," 3GPP, Tech. Rep., Mar. 2017.

[13] 3GPP MCC Support, "Draft Report of 3GPP TSG RAN WG1 \#89 v0.2.0," 3GPP TSG RAN WG1 Meeting \#89, Hangzhou, China, May. 2017.

[14] Ericsson, "R1-1708942. Radio resource pool sharing between mode 3 and mode 4 UEs," 3GPP TSG RAN WG1 Meeting \#89, Hangzhou, China, May. 2017.

[15] 3GPP, "TR 36.885 Study on LTE-based V2X services (v14.0.0, Release 14)," 3GPP, Tech. Rep., Jul. 2016.

[16] Huawei, HiSilicon, "R1-160284. DMRS enhancement of V2V," 3GPP TSG RAN WG1 Meeting \#84, St. Julian's, Malta, Feb. 2016.

[17] M. Sepulcre, J. Gozalvez, B. Coll-Perales "Why 6Mbps is not (always) the Optimum Data Rate for Beaconing in Vehicular Networks", IEEE Transactions on Mobile Computing, Early Access, 2017. 


\section{Acknowledgements}

This work is supported in part by the Spanish Ministerio de Economía y Competitividad and FEDER funds under the project TEC2014-5716-R and research grant PEJ-2014-A33622 .

Rafael Molina-Masegosa (rafael.molinam@umh.es) received a Telecommunications Engineering Degree from the University of Granada (UGR), Spain. During his final degree project, he worked on brain image processing, directed by researchers from Signal Processing and Biomedical Applications (SiPBA) group from the UGR. In December 2015, he joined the UWICORE research laboratory from the Universidad Miguel Hernández de Elche (UMH), Spain, where he is currently conducting his PhD working on $5 G$ wireless vehicular networks.

Javier Gozalvez (j.gozalvez@umh.es) received an electronics engineering degree from the Engineering School ENSEIRB (Bordeaux, France), and a PhD in mobile communications from the University of Strathclyde, Glasgow, U.K. Since October 2002, he is with the Universidad Miguel Hernández de Elche (UMH), Spain, where he is currently an Associate Professor and Director of the UWICORE laboratory. At UWICORE, he leads research activities in the areas of vehicular networks, $5 G$ device-centric networks, and wireless industrial networks. He has published over 135 papers in international conferences and journals, and has been Principal Investigator for 30 research projects and contracts. He has received several awards at international and national conferences, the best research paper award from the Journal of Network and Computer Applications (Elsevier) in 2014, and the Runner-up prize for the "Juan López de Peñalver" award of the Royal Academy of Engineering in Spain that recognizes the most notable Spanish engineers aged below 40. He is an expert evaluator for the European Commission and research agencies across Europe. 